\title{
A Novel Multiscale Physics Based Progressive Failure Methodology for Laminated Composite Structures
}

\author{
Evan J. Pineda* and Anthony M. Waas ${ }^{\dagger}$ \\ University of Michigan, Ann Arbor, MI 48109 \\ Brett A. Bednarcyk ${ }^{\ddagger}$ \\ NASA Glenn Research Center, Cleveland, OH 44135 \\ Craig S. Collier ${ }^{\S}$ and Phillip W. Yarrington ${ }^{\S}$ \\ Collier Research Corporation
}

\begin{abstract}
A variable fidelity, multiscale, physics based finite element procedure for predicting progressive damage and failure of laminated continuous fiber reinforced composites is introduced. At every integration point in a finite element model, progressive damage is accounted for at the lamina-level using thermodynamically based Schapery Theory. Separate failure criteria are applied at either the global-scale or the micro-scale in two different FEM models. A micromechanics model, the Generalized Method of Cells, is used to evaluate failure criteria at the micro-level. The stress-strain behavior and observed failure mechanisms are compared with experimental results for both models.
\end{abstract}

\section{Introduction}

The ability to optimize the design of composite structures is limited by the prediction capabilities of numerous progressive damage and failure analysis methods. In order to utilize the full potential of these methods, a distinction between damage and failure must be established. Failure indicates a global catastrophe, such as macroscopic cracking; therefore, it is an event that leads to large changes in the material properties at the failed material point. Damage, though, leads to a gradual reduction in (not complete absence of) load carrying capability. In most instances, damage reaches a critical state and becomes unstable resulting in failure; however, these mechanisms need to be treated separately. By considering both progressive damage and failure, the response of a carbon fiber laminated composite structure can be more accurately characterized.

Many methods utilize failure criteria, along with linear elasticity, to predict the load carrying capabilities of composite structures. Typically, progressive failure is introduced into finite element simulations by computing load displacement behavior using an elasticity solution until a failure criterion is met locally at an integration point. Upon satisfying this criterion, specific moduli are reduced to negligible values at that integration point. The moduli that are abated are chosen based on the failure mechanism being modeled.

This approach does not account for all the damage mechanisms observed when a composite structure is loaded, particularly matrix microdamage. Matrix microdamage, or microcracking, in epoxy matrix composites is the growth of distributed, microscopic voids and fissures within the matrix, that are consequences of the manufacturing process and the deformation experienced by the polymer. Progressive microdamage is the primary cause of nonlinearity in fiber reinforced laminates (FRLs) up to the onset of failure. ${ }^{1}$ As microdamage accumulates due to mechanical loading, the elastic moduli of the composite are permanently, and progressively, degraded. This damage manifests in the matrix of a composite structure; as a result, only $E_{22}$ and $G_{12}$ of a lamina are significantly affected.

*Ph.D Pre-candidate, Mechanical Engineering Department.

$\dagger$ Professor, Aerospace Engineering Department.

${ }_{\ddagger}$ Materials Research Engineer, AIAA Senior Member.

$\S$ Hypersizer Software, AIAA Senior Member 
Damage progression in a composite leads to a fundamentally different stress-strain response from the material. If the structure contains notches or holes, as is the case for most composite panels in service, localized reduction in moduli leads to redistribution of the stress and strain fields. These fields cannot be captured accurately if a failure criterion is used in conjunction with linear elasticity because the properties in all locations that have not reached failure are assumed uniform.

In a composite structure, the coupled failure of the individual constituents leads to the globally observed failure mechanisms. The failure of the constituents is commonly modeled by reducing the lamina properties that are affected most by the properties of the failed material. This homogenization does not capture the interaction between the constituents, and can lead to a misrepresentation of the failure mechanisms that accrue when the structure is loaded. Micromechanics can be used to resolve the composite into its components. Failure can be evaluated in each of the constituents, thus capturing the interactions due to failure of the individual materials in the composite.

Two new finite element procedures for predicting progressive damage and failure of FRLs were developed. The objective of this paper is to show that, for the laminates studied, the use of macroscopic failure criteria is redundant in view of assigning critical states to the accumulated progressive damage that occur within a lamina.

At every integration point in each finite element model, progressive damage is accounted for at the lamina-level using a thermodynamically based theory developed by Schapery. ${ }^{1}$ Failure criteria are applied at either the global-scale or the micro-scale. Lamina level failure is evaluated via the 2-D Hashin-Rotem failure criterion. $^{2}$ A micromechanics model, the Generalized Method of Cells (GMC) developed by Paley and Aboudi, ${ }^{3}$ is used to evaluate the 3-D Tsai-Hill failure criterion ${ }^{4}$ in the matrix phase at the micro-level, and a maximum stress criterion is utilized for fiber failure. Results from the two different finite element models are examined and compared with experimental data from Bogert et al. ${ }^{5}$ in Section IV.

\section{Multiscale Modeling of Damage and Failure}

\section{II.A. Lamina Level Modeling of Progressive Damage Using Schapery Theory}

\section{II.A.1. Thermodynamically based work potential model}

Progressive damage in the epoxy matrix composite is modeled using Schapery Theory (ST). ${ }^{1}$ This thermodynamics based, work potential theory is capable of capturing the microdamage mechanisms responsible for the material nonlinearity by dividing the total applied work, $W_{T}$ into a recoverable part (elastic), $W$, and a dissipated portion (work of structural change), $W_{S}$.

$$
W_{T}=W+W_{S}
$$

As the material is loaded, a portion of the applied work facilitates structural changes in the material. These structural changes, such as microcracking, affect the elastic properties of the material. A portion of the total applied work is recovered when the structure is unloaded. The magnitude of work recovered is contingent upon the current degraded elastic properties. Upon subsequent reloading, the material will behave linearly, exhibiting the elastic properties observed during unloading, until the material reaches the previous maximum strain state. After this state is achieved, structural changes resume, further degrading the elastic moduli of the material. This process is shown in Figure 1. The shaded area represents $W_{S}$, and the area under the linear unloading curve is $W$. It is assumed that the material behaves as a secant material, a reasonable assumption for FRLs. ${ }^{6}$

Both $W$ and $W_{S}$ are functions of a set of internal state variables (ISVs), $S_{m},(m=1,2, M)$. These ISVs account for any inelastic structural changes in the material. Differentiating $W_{S}$ with respect to any ISV, $S_{m}$ yields the thermodynamic force, $f_{m}$, available for producing structural changes associated with the $m^{t h}$ ISV.

$$
f_{m}=\frac{\partial W_{s}}{\partial S_{m}}
$$

It is shown in Ref. 1 that the total work is at a minimum with respect to each ISV.

$$
\frac{\partial W_{T}}{\partial S_{m}}=0
$$




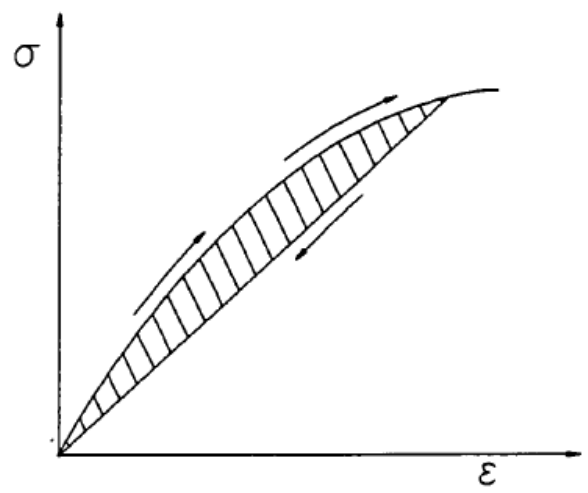

Figure 1. Irrecoverable portion of total work, $W_{S}$, is represented by the shaded area.

Additionally, Rice ${ }^{7}$ has shown that according to the second law of thermodynamics:

$$
f_{m} \dot{S}_{m} \geq 0
$$

Equations (2), (3), and (4) form the foundation of a thermodynamically based work potential model for nonlinear structural changes in a material.

\section{II.A.2. Application of ST to fiber reinforced plastic composites}

Damage accumulates in a composite through numerous mechanisms affecting the constituent materials in the composite. Micro-level damage, which includes matrix microcracking, fiber kinking and debonding, is a class of damage mechanisms separate from matrix failure due to transverse (macroscopic) cracking. Matrix damage accumulates gradually at the micro-level until its effects are superceded by the rapid progression of macroscopic cracks. Like matrix macrocracking, fiber damage also does not typically occur progressively, but rather, abruptly. Moreover, once the fibers in a composite lamina begin to break, it has nearly lost its entire load carrying capabilities. Across the breakline, however, the adjacent layers carry the loads through load re-distribution. Unfortunately, microdamage mechanisms are often overlooked in analyses; instead, the more catastrophic matrix macrocracking and fiber breakage are the focus. ST is capable of modeling the effects of progressive microdamage in the matrix phase of FRLs.

The inelastic work of structural change, $W_{S}$, can be a function of any number of state variables. To apply this work potential model to progressive failure analysis, it is assumed that the structural changes which result from microdamage, depend on only one ISV, $S$. This ISV is assumed responsible for all material nonlinearities up to macroscopic matrix cracking (global cracking), delamination and fiber breakage, and account for all damage present in the matrix of each lamina in a composite structure.

It can be assumed that $W_{S}$ is an additive function of the ISVs, $W_{S}=\sum_{i}^{m} W_{i}\left(S_{i}\right)$. Furthermore $W_{i}$ are in one-to-one correspondence with their arguments; so, $W_{i}$ can be chosen such that $W_{i}=S_{i}$. Since, in this case, $W_{S}$ is a function of only one ISV, $W_{S}$ can be chosen such that ISV, $W_{S}=S$. Therefore, the ISV governing the amount of work used to advance microdamage and that actual work are equivalent. Equation (1) can now be recast.

$$
W_{T}=W+S
$$

Differentiating (5) with respect to $S$, and utilizing Equation (3) yields:

$$
\frac{\partial W}{\partial S}=-1
$$

Additionally, combining Equations (2) and (4) with $W_{S}=S$ results in

$$
\dot{S} \geq 0
$$

which is a statement on the inadmissibility of damage "healing". Equation (7) dictates that the amount of work used to progress microdamage can never decrease; therefore, that energy has been dissipated into creating structural change and cannot be recovered. The combination of Equations (6) and (7) represent the evolution equations for microdamage in the matrix of the composite. 


\section{II.A.3. Formulation of constitutive law}

Plane stress lamina stress-strain relationships can be written in principal material coordinates as

$$
\begin{aligned}
& \sigma_{11}=Q_{11} \epsilon_{11}+Q_{12} \epsilon_{22} \\
& \sigma_{22}=Q_{12} \epsilon_{11}+Q_{22} \epsilon_{22} \\
& \tau_{12}=Q_{66} \gamma_{12}
\end{aligned}
$$

where $\gamma_{12}$ is the engineering shear strain and

$$
\begin{aligned}
Q_{11} & =\frac{E_{11}}{1-\nu_{12} \nu_{21}} \\
Q_{22} & =\frac{E_{22}}{1-\nu_{12} \nu_{21}} \\
Q_{12} & =\nu_{12} Q_{22} \\
Q_{66} & =G_{12} \\
\nu_{21} & =\frac{\nu_{12} E_{22}}{E_{11}}
\end{aligned}
$$

where $E_{11}$ is the axial elastic modulus, $E_{22}$ is the transverse elastic modulus, $\nu_{12}$ is the Poisson's Ratio, $\nu_{21}$ is the transverse Poisson's Ratio and $G_{12}$ is the elastic shear modulus. After assuming that the quantity $\nu_{12} \nu_{21}<<1$, Equations (9) become,

$$
\begin{aligned}
& Q_{11}=E_{11} \\
& Q_{22}=E_{22} \\
& Q_{12}=\nu_{12} Q_{22} \\
& Q_{66}=G_{12}
\end{aligned}
$$

\section{II.A.4. Determining the damage state}

It is necessary to define in what manner the moduli degrade as functions of the ISV. Since the damage mechanisms considered in this progressive damage approach are exclusive to the matrix of the composite, it is safe to assume that the moduli affected by this damage are limited to $E_{22}$ and $G_{12}$. These moduli can be written as functions of $S$.

$$
\begin{aligned}
E_{22} & =E_{220} e_{s}(S) \\
G_{12} & =G_{120} g_{s}(S)
\end{aligned}
$$

where $E_{220}$ and $G_{120}$ are the undamaged transverse and shear elastic moduli, $e_{s}(S)$ and $g_{s}(S)$ are factors relating the transverse and shear moduli to the microdamage, $S$. Sicking ${ }^{6}$ provided a procedure for determining $e_{s}$ and $g_{s}$ experimentally. The experimental curves can then be fit with polynomials (such that moduli at $S=0$ are $E_{22}=E_{220}$ and $G_{12}=G_{120}$, corresponding to an undamaged state) and used in Equations (11) and (12).

The elastic strain energy density, $W$, can be written using the constitutive relationships.

$$
W=\frac{1}{2}\left(E_{11} \epsilon_{11}^{2}+E_{22} \epsilon_{22}^{2}+G_{12} \gamma_{12}^{2}\right)+Q_{12} \epsilon_{11} \epsilon_{22}
$$

Employing Equation (6) with (11), (12), and (13), and assuming the quantity $Q_{12}=\nu_{12} Q_{22}$ is constant and independent of $S$, yields a damage evolution ordinary differential equation which can be solved for $S$.

$$
\frac{\epsilon_{22}^{2}}{2} \frac{\partial E_{22}}{\partial S}+\frac{\gamma_{12}^{2}}{2} \frac{\partial G_{12}}{\partial S}=-1
$$

The above equation indicates that the work of structural change depends only on the strain state, the initial virgin composite moduli ( $E_{220}$ and $\left.G_{120}\right)$, and the damage functions $\left(e_{s}\right.$ and $\left.g_{s}\right)$.

Experimentally it has been determined that $\mathrm{S}$ behaves as $\epsilon^{3}$, thus it is convenient to introduce a reduced damage variable, $S_{r}$.

$$
S_{r} \equiv S^{1 / 3}
$$


Using the reduced ISV the evolution equation, Equation (14), becomes

$$
\frac{\epsilon_{22}^{2}}{2} \frac{\partial E_{22}}{\partial S_{r}}+\frac{\gamma_{12}^{2}}{2} \frac{\partial G_{12}}{\partial S_{r}}=-3 S_{r}^{2}
$$

Once $S$ is determined from Equation (16), the transverse and shear moduli can be degraded accordingly using Equations (11) and (12).

In previous studies, ${ }^{8}$ where the loading was compression dominated, the instantaneous fiber rotation is captured in conjunction with ST. This has the advantage of predicting fiber kinking failure, avoiding the use of an explicit fiber direction compressive strength criterion (see Equation (45) later). In the present work, where loading is considered tension dominated, the effect of including the fiber rotation was initially deemed unnecessary. However, the discoveries reported in Section IV.B indicate that including this feature would also be worthwhile for this problem.

\section{II.B. Micromechanical Modeling Using the Generalized Method of Cells}

A micromechanical analysis technique, coined the Method of Cells, was developed by Aboudi; ${ }^{9}$ subsequently, Paley and Aboudi $^{3}$ expanded the Method of Cells into the Generalized Method of Cells (GMC), and later Aboudi et al. ${ }^{10}$ further increased the robustness of this method with the High Fidelity Method of Cells (HFGMC). These methods provide semi-closed form solutions for determining global anisotropic composite properties in terms of the constituent materials, as well as, stresses and strains in each of the constituent subcells. The sophisticated methods (GMC and HFGMC) offer a high degree of accuracy at a relatively low computational cost. The following sections detail the formulation of GMC (employed herein). The reader is referred to Ref. 10 for details on HFGMC.

\section{II.B.1. Kinematics and constitutive relationships}

It is assumed that a unidirectional fiber composite can be represented as a collection of repeating volume elements (RVE). Paley and Aboudi ${ }^{3}$ chose to model this RVE as an element consisting of $N_{\beta} \mathrm{x} N_{\gamma}(\beta, \gamma=$ $\left.1,2, N_{\beta, \gamma}\right)$ subcells as shown in Figure 2. Each of these subcells is occupied by one of the constituents in the

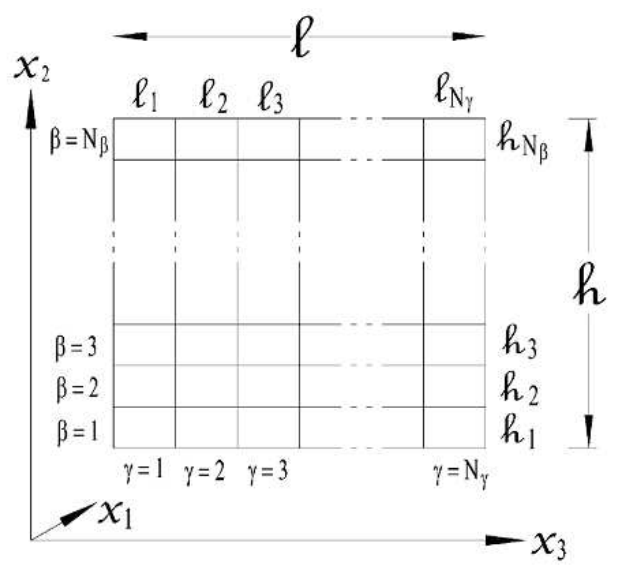

Figure 2. Representative volume element used in GMC. ${ }^{3}$

composite. The number of subcells and the materials occupying each subcell is completely general. For a two-phase fibrous composite any desired micro-structure can be represented by occupying each subcell with either a matrix or fiber constituent.

The $x_{1}$-axis shown in Figure 2 is the fiber direction, and the cross-sectional area of each subcell is given by $h_{\beta} \ell_{\gamma}$. A local coordinate system $\left(x_{1}, \bar{x}_{2}^{(\beta)}, \bar{x}_{3}^{(\gamma)}\right)$ can be introduced with its origin located at the center of each subcell, as shown in Figure 3. The objective of this method is to determine the average behavior of the composite material; thus, it is sufficient to model the displacements in each subcell using a linear theory (HFGMC employs a higher order displacement approximation).

$$
u_{i}^{(\beta \gamma)}=w_{i}^{(\beta \gamma)}+\bar{x}_{2}^{(\beta)} \phi_{i}^{(\beta \gamma)}+\bar{x}_{3}^{(\gamma)} \psi_{i}^{(\beta \gamma)}
$$




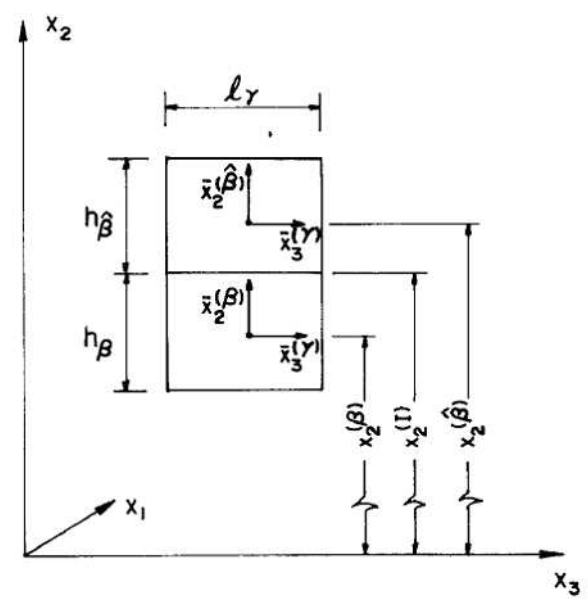

Figure 3. Local coordinates used in GMC subcells. ${ }^{3}$

where $i=1,2,3, w_{i}^{(\beta \gamma)}$ is the displacement at the center of subcell $\beta \gamma$. Microvariables $\left(\phi_{i}^{(\beta \gamma)}, \psi_{i}^{(\beta \gamma)}\right)$ characterize the first-order dependence of the displacement field on the local coordinates $\bar{x}_{2}^{(\beta)}$ and $\bar{x}_{3}^{(\gamma)}$.

The components of the strain tensor follow from Equation (17) as,

$$
\epsilon_{i j}^{(\beta \gamma)}=\frac{1}{2}\left(\partial_{i} u_{j}^{(\beta \gamma)}+\partial_{j} u_{i}^{(\beta \gamma)}\right)
$$

where $\partial_{1}=\frac{\partial}{\partial x_{1}}, \partial_{2}=\frac{\partial}{\partial \bar{x}_{2}^{(\beta)}}, \partial_{3}=\frac{\partial}{\partial \bar{x}_{3}^{(\gamma)}}$. Substituting Equation (17) into Equation (18) results in the six components of the average strain tensor for each subcell in terms of the microvariables.

$$
\begin{aligned}
& \bar{\epsilon}_{11}^{(\beta \gamma)}=\partial_{1} w_{1}^{(\beta \gamma)} \\
& \bar{\epsilon}_{22}^{(\beta \gamma)}=\phi_{2}^{(\beta \gamma)} \\
& \bar{\epsilon}_{33}^{(\beta \gamma)}=\psi_{3}^{(\beta \gamma)} \\
& 2 \bar{\epsilon}_{23}^{(\beta \gamma)}=\phi_{3}^{(\beta \gamma)}+\psi_{2}^{(\beta \gamma)} \\
& 2 \bar{\epsilon}_{13}^{(\beta \gamma)}=\psi_{1}^{(\beta \gamma)}+\partial_{1} w_{3}^{(\beta \gamma)} \\
& 2 \bar{\epsilon}_{12}^{(\beta \gamma)}=\phi_{1}^{(\beta \gamma)}+\partial_{1} w_{2}^{(\beta \gamma)}
\end{aligned}
$$

The constitutive law also needs to be defined for each subcell, and can use any stress-strain relationship desired. In this work, it will be assumed that only elastic subcell strains, $\bar{\epsilon}_{i j}^{(\beta \gamma)}$, are present. However, the constitutive law can be amended to incorporate additional strains such as thermal and inelastic strains. Hooke's law for relating the subcell stresses, $\bar{\sigma}_{i j}^{(\beta \gamma)}$, to the elastic subcell strains can be written as

$$
\bar{\sigma}_{i j}^{(\beta \gamma)}=C_{i j k l}^{(\beta \gamma)} \bar{\epsilon}_{k l}^{(\beta \gamma)}
$$

where $C_{i j k l}^{(\beta \gamma)}$ are the components of the elastic stiffness tensor.

\section{II.B.2. Displacement continuity conditions}

It is required that subcell displacements are continuous at the interfaces between adjacent subcells, as well as at the boundaries between neighboring repeating cells. Enforcing these conditions will yield $2\left(N_{\beta}+\right.$ $\left.N_{\gamma}\right)+N_{\beta} N_{\gamma}+1$ equations. Moreover, all microvariables are eliminated from these equations. For detailed derivations of displacement and traction continuity conditions see Ref. 3. 
The first necessary equation is obtained by defining the average strains in the composite, $\bar{\epsilon}_{i j}$, in terms of the average subcell strains.

$$
\bar{\epsilon}_{i j}=\frac{1}{h \ell} \sum_{\beta=1}^{N_{\beta}} \sum_{\gamma=1}^{N_{\gamma}} h_{\beta} \ell_{\gamma} \bar{\epsilon}_{i j}^{(\beta \gamma)}
$$

where $h, \ell$ and $h_{\beta}, \ell_{\gamma}$ represent the repeating cell, and subcell geometry, respectively (see Figures 2 and 3 ).

Next, displacement continuity is satisfied in an average sense over all subcell and repeating cell interfaces. Since these continuity conditions are satisfied on average, the shape of the fiber does not appear in the final result. Thus, no stress concentrations are developed at the corners, and the end result is that the subcell strains, and stresses, are determined as a function of only the fiber volume fraction and constituent properties.

After enforcing average displacement continuity on the interfaces, the following $2\left(N_{\beta}+N_{\gamma}\right)$ equations are produced:

$$
\begin{array}{ll}
\sum_{\beta=1}^{N_{\beta}} h_{\beta} \bar{\epsilon}_{22}^{(\beta \gamma)}=h \bar{\epsilon}_{22}, & \gamma=1, \ldots, N_{\gamma} \\
\sum_{\gamma=1}^{N_{\gamma}} \ell_{\gamma} \bar{\epsilon}_{33}^{(\beta \gamma)}=\ell \bar{\epsilon}_{33}, & \beta=1, \ldots, N_{\beta} \\
\sum_{\beta=1}^{N_{\beta}} h_{\beta} \bar{\epsilon}_{12}^{(\beta \gamma)}=h \bar{\epsilon}_{12}, & \gamma=1, \ldots, N_{\gamma} \\
\sum_{\gamma=1}^{N_{\gamma}} \ell_{\gamma} \bar{\epsilon}_{13}^{(\beta \gamma)}=\ell \bar{\epsilon}_{13}, & \beta=1, \ldots, N_{\beta}
\end{array}
$$

The final $N_{\beta} N_{\gamma}$ equations come from enforcing uniform strain in the $x_{1}$-direction across all subcells.

$$
\bar{\epsilon}_{11}^{(\beta \gamma)}=\bar{\epsilon}_{11}
$$

Equations (22) - (26) can be rearranged and rewritten in matrix from.

$$
\mathbf{A}_{G} \epsilon_{S}=\mathbf{J} \bar{\epsilon}
$$

where

$$
\bar{\epsilon}=\left\{\bar{\epsilon}_{11}, \bar{\epsilon}_{22}, \bar{\epsilon}_{33}, 2 \bar{\epsilon}_{23}, 2 \bar{\epsilon}_{13}, 2 \bar{\epsilon}_{12}\right\}
$$

and

$$
\epsilon_{S}=\left[\bar{\epsilon}^{(11)}, \bar{\epsilon}^{(12)}, \ldots, \bar{\epsilon}^{\left(N_{\beta} N_{\gamma}\right)}\right]
$$

where $\bar{\epsilon}^{(\beta \gamma)}$ are vectors containing the average subcell strains in the same order as Equation (28).

\section{II.B.3. Traction continuity conditions}

Traction continuity must also be enforced in order to arrive at the correct number of equations needed to solve for the $6 N_{\beta} N_{\gamma}$ subcell strain unknowns. However, some of the traction continuity conditions are redundant. After eliminating repeating traction conditions, the following $5 N_{\beta} N_{\gamma}-2\left(N_{\beta}+N_{\gamma}\right)-1$ independent equations remain:

$$
\begin{array}{ll}
\bar{\sigma}_{22}^{(\beta \gamma)}=\bar{\sigma}_{22}^{(\hat{\beta} \gamma)}, & \beta=1, \ldots, N_{\beta}-1, \quad \gamma=1, \ldots, N_{\gamma} \\
\bar{\sigma}_{33}^{(\beta \gamma)}=\bar{\sigma}_{33}^{(\beta \hat{\gamma})}, & \beta=1, \ldots, N_{\beta}, \quad \gamma=1, \ldots, N_{\gamma}-1 \\
\bar{\sigma}_{23}^{(\beta \gamma)}=\bar{\sigma}_{23}^{(\hat{\beta} \gamma)}, & \beta=1, \ldots, N_{\beta}-1, \quad \gamma=1, \ldots, N_{\gamma} \\
\bar{\sigma}_{32}^{(\beta \gamma)}=\bar{\sigma}_{32}^{(\beta \hat{\gamma})}, & \beta=1, \ldots, N_{\beta}, \quad \gamma=1, \ldots, N_{\gamma}-1 \\
\bar{\sigma}_{21}^{(\beta \gamma)}=\bar{\sigma}_{21}^{(\hat{\beta} \gamma)}, & \beta=1, \ldots, N_{\beta}-1, \quad \gamma=1, \ldots, N_{\gamma}
\end{array}
$$




$$
\bar{\sigma}_{31}^{(\beta \gamma)}=\bar{\sigma}_{31}^{(\beta \hat{\gamma})}, \quad \beta=1, \ldots, N_{\beta}, \quad \gamma=1, \ldots, N_{\gamma}-1
$$

where $\hat{\beta}$ and $\hat{\gamma}$ are given by

$$
\begin{aligned}
& \hat{\beta}= \begin{cases}\beta+1, & \beta<N_{\beta} \\
1, & \beta=N_{\beta}\end{cases} \\
& \hat{\gamma}= \begin{cases}\gamma+1, & \gamma<N_{\gamma} \\
1, & \gamma=N_{\gamma}\end{cases}
\end{aligned}
$$

These traction conditions can be recast in terms of the average subcell strains using the constitutive relationship, in this case Equation (20). The equations can then be rewritten in matrix form.

$$
\mathbf{A}_{M} \epsilon_{S}=\mathbf{0}
$$

where $\epsilon_{S}$ is given in Equation (29)

\section{II.B.4. Determining subcell strains}

Once $\mathbf{A}_{G}, \mathbf{A}_{M}$, and $\mathbf{J}$ have been determined, the subcell strains can be computed by solving

$$
\tilde{\mathbf{A}} \epsilon_{S}=\mathbf{K} \bar{\epsilon}
$$

where

$$
\tilde{\mathbf{A}}=\left[\begin{array}{c}
\mathbf{A}_{M} \\
\mathbf{A}_{G}
\end{array}\right]
$$

and

$$
\mathbf{K}=\left[\begin{array}{l}
\mathbf{0} \\
\mathbf{J}
\end{array}\right]
$$

After the subcell strains are obtained, it is trivial to produce the subcell stresses using the constitutive law.

\section{II.C. Failure Criteria}

\section{II.C.1. Modeling lamina-level failure with Hashin-Rotem failure criteria}

In this work, failure is treated separately from damage. Damage is considered the progressive deterioration of the matrix. A method for modeling damage was presented in Section II.A. Failure, however, is a localized, catastrophic event, after which the material can no longer support any load in a particular direction at the point of failure.

There are numerous theories in the literature that offer methods for evaluating the onset of catastrophic failure. One criterion used in this investigation is the 2-D Hashin-Rotem (H-R) failure theory, ${ }^{2}$ which is also used in Ref. 5. This lamina theory is the simplest, empirical theory that still retains the transversely isotropic nature of unidirectional fiber composites.

Four separate criteria encompass the H-R failure theory. Transverse (perpendicular to the fiber direction) and shear stresses are responsible for matrix failure in a unidirectional fiber composite. Matrix failure at any material point is dictated by

$$
\left(\frac{\sigma_{22}}{Y_{t}}\right)^{2}+\left(\frac{\tau_{12}}{T}\right)^{2}=d_{m}^{2}, \quad \sigma_{22}>0
$$

when the transverse stresses, $\sigma_{22}$, are greater than zero (tension), and

$$
\left(\frac{\sigma_{22}}{Y_{c}}\right)^{2}+\left(\frac{\tau_{12}}{T}\right)^{2}=d_{m}^{2}, \quad \sigma_{22}<0
$$

when $\sigma_{22}$ is less than zero (compression), where $\tau_{12}$ is the shear stress in the composite material coordinates, $Y_{t}$ is the matrix transverse strength in tension, $Y_{c}$ is the matrix transverse strength in compression, and $T$ is 
the matrix shear strength. Once $d_{m}$ is greater than or equal to one, that matrix at that location has failed, and that point can no longer sustain transverse or shear loads. Although, it is assumed that such a point will still be able to carry loads in the fiber direction until it reaches the limit of fiber failure.

A similar set of criteria is used to govern fiber failure. When the stress in the fiber direction, $\sigma_{11}$, is greater than zero, the fiber failure criterion used is

$$
\left(\frac{\sigma_{11}}{X_{t}}\right)^{2}=d_{f}^{2}, \quad \sigma_{11}>0
$$

Whereas, when the fibers in the composite are subjected to a compressive load $\left(\sigma_{11}<0\right)$

$$
\left(\frac{\sigma_{11}}{X_{c}}\right)^{2}=d_{f}^{2}, \quad \sigma_{11}<0
$$

is used. The fiber direction tensile strength is $X_{t}$, and $X_{c}$ is the compressive strength. Fiber failure occurs when $d_{f} \geq 1$, after which, that point can no longer carry axial loads.

The H-R failure criterion provides a simple 2-D model for predicting localized failure in a finite element model. However, as is mentioned in Ref. 5, the predictions of the failure criteria are dependent on the density of the finite element mesh near a notch tip. This indicates that new stress measures that incorporate the length scales associated with the discretization process needs to be developed in order to obtain a robust prediction model. ${ }^{11}$

\section{II.C.2. Modeling micro-level constituent failure with Tsai-Hill}

The multiscale computational method (MCM) employed in one of the FEM simulations evaluated uses GMC to resolve the applied global stresses to the micro-constituent level. Failure of the matrix phase is established using the 3-D Tsai-Hill failure criterion. ${ }^{4}$ Assuming failure in the matrix subcells is isotropic, the criterion is given as

$$
\begin{aligned}
& \frac{\left.\left(\bar{\sigma}_{11}^{\left(\beta_{m} \gamma_{m}\right.}\right)\right)^{2}+\left(\bar{\sigma}_{22}^{\left(\beta_{m} \gamma_{m}\right)}\right)^{2}+\left(\bar{\sigma}_{33}^{\left(\beta_{m} \gamma_{m}\right)}\right)^{2}}{Y_{m t}^{2}} \\
& \quad+\frac{-\bar{\sigma}_{11}^{\left(\beta_{m} \gamma_{m}\right)} \bar{\sigma}_{22}^{\left(\beta_{m} \gamma_{m}\right)}-\bar{\sigma}_{11}^{\left(\beta_{m} \gamma_{m}\right)} \bar{\sigma}_{33}^{\left(\beta_{m} \gamma_{m}\right)}-\bar{\sigma}_{22}^{\left(\beta_{m} \gamma_{m}\right)} \bar{\sigma}_{33}^{\left(\beta_{m} \gamma_{m}\right)}}{Y_{m t}^{2}} \\
& \quad+\frac{\left(\bar{\sigma}_{12}^{\left(\beta_{m} \gamma_{m}\right)}\right)^{2}+\left(\bar{\sigma}_{13}^{\left(\beta_{m} \gamma_{m}\right)}\right)^{2}+\left(\bar{\sigma}_{23}^{\left(\beta_{m} \gamma_{m}\right)}\right)^{2}}{T_{m}^{2}}=d_{m}^{2}, \quad \bar{\sigma}_{22}>0
\end{aligned}
$$

where $\beta_{m}$ and $\gamma_{m}$ are the matrix subcell indices and the average applied transverse stress, $\bar{\sigma}_{22}$, is tensile. Similarly, for applied compressive transverse stresses:

$$
\begin{aligned}
& \frac{\left.\left(\bar{\sigma}_{11}^{\left(\beta_{m} \gamma_{m}\right.}\right)\right)^{2}+\left(\bar{\sigma}_{22}^{\left(\beta_{m} \gamma_{m}\right)}\right)^{2}+\left(\bar{\sigma}_{33}^{\left(\beta_{m} \gamma_{m}\right)}\right)^{2}}{Y_{m c}^{2}} \\
& \quad+\frac{-\bar{\sigma}_{11}^{\left(\beta_{m} \gamma_{m}\right)} \bar{\sigma}_{22}^{\left(\beta_{m} \gamma_{m}\right)}-\bar{\sigma}_{11}^{\left(\beta_{m} \gamma_{m}\right)} \bar{\sigma}_{33}^{\left(\beta_{m} \gamma_{m}\right)}-\bar{\sigma}_{22}^{\left(\beta_{m} \gamma_{m}\right)} \bar{\sigma}_{33}^{\left(\beta_{m} \gamma_{m}\right)}}{Y_{m c}^{2}} \\
& \quad+\frac{\left(\bar{\sigma}_{12}^{\left(\beta_{m} \gamma_{m}\right)}\right)^{2}+\left(\bar{\sigma}_{13}^{\left(\beta_{m} \gamma_{m}\right)}\right)^{2}+\left(\bar{\sigma}_{23}^{\left(\beta_{m} \gamma_{m}\right)}\right)^{2}}{T_{m}^{2}}=d_{m}^{2}, \quad \bar{\sigma}_{22}<0
\end{aligned}
$$

where $Y_{m t}$ and $Y_{m c}$ are the matrix transverse tensile and compressive strengths, respectively, and $T_{m}$ is the matrix shear strength.

Maximum stress criteria, analogous to Equations (44) and (45), are used to dictate failure in the fiber subcells.

$$
\begin{aligned}
& \left(\frac{\bar{\sigma}_{11}^{\left(\beta_{f} \gamma_{f}\right)}}{X_{f t}}\right)^{2}=d_{f}^{2}, \quad \bar{\sigma}_{11}>0 \\
& \left(\frac{\bar{\sigma}_{11}^{\left(\beta_{f} \gamma_{f}\right)}}{X_{f c}}\right)^{2}=d_{f}^{2}, \quad \bar{\sigma}_{11}<0
\end{aligned}
$$


where $\beta_{f}$ and $\gamma_{f}$ are the fiber subcell indices, $X_{f t}$ and $X_{f c}$ are the tensile and compressive fiber strengths, and $\bar{\sigma}_{11}$ is the applied axial stress.

Failure arises in a matrix subcell when $d_{m} \geq 0$ and in a fiber subcell when $d_{f} \geq 0$ for that subcell. Once a subcell has failed, all the properties of that subcell are degraded appropriately.

\section{Finite Element Model Description}

Two different finite element models were used to predict the behavior of a notched carbon fiber reinforced epoxy panel first introduced in Ref. 5. Two laminate stacking sequences, shown in Table 1, were modeled, and the elastic properties corresponding to T800/3900-2 (Table 2) were used as the initial properties for each layer.

\begin{tabular}{|l|c|c|}
\hline ID & Stacking Sequence & Thickness (in.) \\
\hline \hline Laminate Sequence-1 & {$[0]_{12}$} & 0.078 \\
\hline Laminate Sequence-2 & {$[45 / 0 /-45 / 0 / 90]_{S}$} & 0.065 \\
\hline
\end{tabular}

Table 1. Laminate stacking sequences investigated.

\begin{tabular}{|l|c|}
\hline Property & Value \\
\hline \hline$E_{11}(\mathrm{Msi})$ & 23.2 \\
\hline$E_{22}(\mathrm{Msi})$ & 1.3 \\
\hline$G_{12}(\mathrm{Msi})$ & 0.9 \\
\hline$\nu_{12}$ & 0.28 \\
\hline
\end{tabular}

Table 2. Initial elastic properties of T800/3900-2 lamina.

The mesh used in these FEM models, shown in Figure 4, consists of 6642 nodes and 6480 Abaqus S4R elements. This mesh was used because the high density of elements near the notch tips is needed to produce accurate stress fields at those locations. ${ }^{12}$

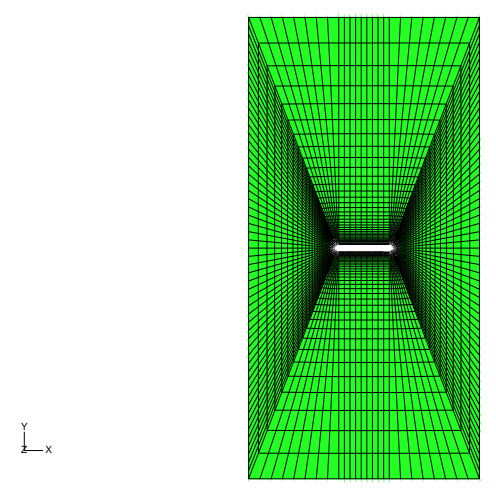

(a) FEM Mesh used in simulations.

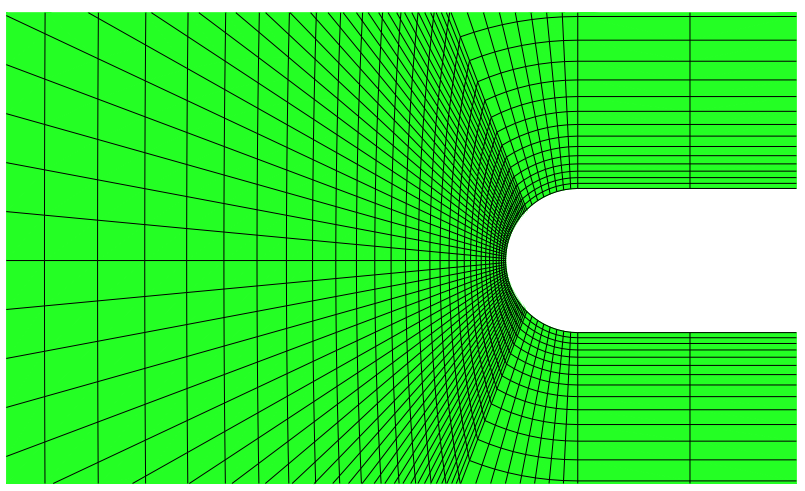

(b) Enlarged view of mesh near notch tip

Figure 4.

No constraints were placed on the vertical edges of the model, and the bottom edge was restricted from moving in the $x, y, z$, and all rotational degrees of freedom. The top edge is fixed in the $x$, and $z$ displacements and all rotations. A vertical displacement is applied in the $y$ direction to simulate tensile loading.

Static analysis is performed in Abaqus/Standard, and an edge displacement of 0.025 inches and 0.065 inches is applied to laminate stacking sequence 1 and 2 , respectively. The maximum allowable displacement in each time step was set to $0.1 \%$, and the minimum allowed displacement was $0.1 \mathrm{E}-7 \%$.

The coupled microdamage-failure models were implemented using the Abaqus user subroutine, UMAT. ${ }^{13}$ Both models use strain to calculate the reduced damage state, $S_{r}$, with Equation (16). The damage functions, 
$e_{s}$ and $g_{s}$ given in Equations (11) and (12) were modeled as fourth order polynomials.

$$
\begin{aligned}
& e_{s}=e_{s 0}+e_{s 1} S_{r}+e_{s 2} S_{r}^{2}+e_{s 3} S_{r}^{3}+e_{s 4} S_{r}^{4} \\
& g_{s}=g_{s 0}+g_{s 1} S_{r}+g_{s 2} S_{r}^{2}+g_{s 3} S_{r}^{3}+g_{s 4} S_{r}^{4}
\end{aligned}
$$

The polynomial coefficients in Equations (50) and (51) are given in Table 3. These values were obtained by scaling the values reported in Ref. ${ }^{6}$ by the ratio of the respective virgin elastic moduli.

\begin{tabular}{|c|c|c|c|}
\hline$E_{22}$ coefficients & Values & $G_{12}$ coefficients & Values \\
\hline \hline$e_{s 0}$ & 1.0000 & $g_{s 0}$ & 1.0000 \\
\hline$e_{s 1}$ & -0.0351 & $g_{s 1}$ & -0.0377 \\
\hline$e_{s 2}$ & -0.0096 & $g_{s 2}$ & -0.0237 \\
\hline$e_{s 3}$ & -0.0016 & $g_{s 3}$ & 0.0053 \\
\hline$e_{s 4}$ & 0.0003 & $g_{s 4}$ & -0.0004 \\
\hline
\end{tabular}

Table 3. Microdamage polynomial coefficients for $E_{22}$ and $G_{12}$.

It is possible that the ratio of $G_{12} / E_{22}$, as calculated by Equations (11) and (12), reaches a value that produces an ill-conditioned Jacobian in the FEM solution. Therefore, $G_{12}$ is restricted from falling below $40 \%$ of $G_{120}$, which corresponds to $S_{r}=3.74 \mathrm{psi}^{\frac{1}{3}}$. After any point reaches this state, $S_{r}$ is still calculated but it is not used to update $G_{12}$ nor $E_{22}$. Exploration into methods to avoid this instability and still allow damage to progress are currently underway. However, the possibility that this numerical instability is signaling a critical damaged state is also being investigated.

\section{III.A. Method 1 - Schapery Theory with Lamina Level Hashin-Rotem (ST/H-R)}

The first algorithm employs the H-R failure criteria at the lamina level. At each material point, the failure criteria are evaluated using the properties in Table 4. All failure strengths were taken directly from Ref. 5,

\begin{tabular}{|l|l|}
\hline Property & Value (Msi) \\
\hline \hline$Y_{t}$ & 0.00872 \\
\hline$Y_{c}$ & 0.0243 \\
\hline$T$ & 0.0048 \\
\hline$X_{t}$ & 0.412 \\
\hline$X_{c}$ & 0.225 \\
\hline
\end{tabular}

Table 4. Hashin-Rotem failure strengths.

except the shear strength, $T$. The shear strength was adjusted to account for the shear modulus degradation resulting from the progressive damage in the laminate. The value that yielded the best results for laminate sequence-1 was used for both laminates.

If Equation (42) or (43) is satisfied, $E_{22}, G_{12}$, and $\nu_{12}$ are reduced to $35 \%$ of their current values. Each subsequent time step, the calculations involving ST are circumvented and $E_{22}, G_{12}$ and $\nu_{12}$ are further reduced by $35 \%$ until $E_{22}$ and $G_{12}$ fall below 1000 psi, and $\nu_{12}$ is less than 0.001 .

After matrix failure has occurred, Equations (44) and (45) are still evaluated to check for fiber failure. Once either of those criteria are satisfied, $E_{11}$ is also reduced to $35 \%$ then reduced by the same percentage in each following time step until it falls below 10,000 psi.

\section{III.B. Method 2 - Schapery Theory with Micro-level Tsai-Hill (ST/GMC/T-H)}

The second method for modeling damage coupled with failure follows the same procedure for determining the damage in the structure as the previous method; however, the failure criteria is no longer evaluated at the lamina level. Instead, the MAC/GMC suite of micro-mechanics codes is used. ${ }^{14,15}$ At each material point, MAC/GMC is called and a $2 \times 2$ RVE, as shown in Figure 5, is used to model that point. The RVE consists of three matrix subcells and one fiber subcell. The fiber and matrix constituents have the initial properties given in Table 5. To provide global properties consistent with those given in Table 2 anisotropic 


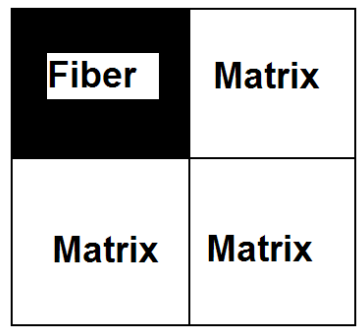

Figure 5. 2 × 2 RVE used in micromechanics simulations.

\begin{tabular}{|l|c|l|c|}
\hline Fiber Properties & Values & Matrix Properties & Values \\
\hline \hline$E_{11}^{f}(\mathrm{Msi})$ & 42.49 & $E_{11}^{m}(\mathrm{Msi})$ & 0.3415 \\
\hline$E_{22}^{f}(\mathrm{Msi})$ & 13.2 & $E_{22}^{m}(\mathrm{Msi})$ & 0.3415 \\
\hline$\nu_{12}^{f}$ & 0.2316 & $\nu_{12}^{m}$ & 0.35 \\
\hline$\nu_{21}^{f}$ & 0.45 & $\nu_{21}^{m}$ & 0.35 \\
\hline$G_{12}^{f}(\mathrm{Msi})$ & 8.0 & $G_{12}^{m}(\mathrm{Msi})$ & 0.3267 \\
\hline
\end{tabular}

Table 5. Elastic properties of fiber and matrix constituents used in GMC.

properties were used for the constituent cells.

At every time step, the micromechanics model must produce composite moduli that are consistent with the lamina level moduli calculated using Equations (11) and (12). Therefore, it is necessary that the moduli of the matrix constituents degrade in a manner that produces consistent $E_{22}$, and $G_{12}$ values. Two fourth order polynomials are used to calculate the matrix Young's Modulus and shear modulus, $E_{m}$ and $G_{m}$, as a function of $S_{r}$.

$$
\begin{aligned}
& E_{m}=E_{m 0}\left(e_{m 0}+e_{m 1} S_{r}+e_{m 2} S_{r}^{2}+e_{m 3} S_{r}^{3}+e_{m 4} S_{r}^{4}\right) \\
& G_{m}=G_{m 0}\left(g_{m 0}+g_{m 1} S_{r}+g_{m 2} S_{r}^{2}+g_{m 3} S_{r}^{3}+g_{m 4} S_{r}^{4}\right)
\end{aligned}
$$

where $E_{m 0}$ and $G_{m 0}$ are the undamaged matrix stiffnesses, and the polynomial coefficients are given in Table 6 . The matrix Poisson's Ratio, $\nu_{m}$, remains unchanged.

\begin{tabular}{|c|r|c|r|}
\hline$E_{m}$ coefficients & Values & $G_{m}$ coefficients & Values \\
\hline \hline$e_{m 0}$ & 1.0000 & $g_{m 0}$ & 1.0000 \\
\hline$e_{m 1}$ & -0.0378 & $g_{m 1}$ & -0.0918 \\
\hline$e_{m 2}$ & -0.0128 & $g_{m 2}$ & -0.0560 \\
\hline$e_{m 3}$ & -0.0005 & $g_{m 3}$ & 0.0137 \\
\hline$e_{m 4}$ & 0.0002 & $g_{m 4}$ & -0.0011 \\
\hline
\end{tabular}

Table 6. Microdamage polynomial coefficients for $E_{22}$ and $G_{12}$.

Using consistent properties for the matrix modulus, the subcell stresses are calculated. Using these stresses, micro-level failure criteria are evaluated. Since the micromechanics model is 3-D, the 2-D H-R failure criteria are no longer applicable. Instead, 3-D Tsai-Hill $(\mathrm{T}-\mathrm{H})$ failure criteria are used for the matrix subcells

The constants used in Equations (46)-(49) are given in Table 7. The matrix shear strength, $T_{m}$, was obtained by applying a global shear stress, $\bar{\sigma}_{12}$, to the RVE. The resulting largest matrix subcell shear stress, $\bar{\sigma}_{12}^{(12)}$ is used as the matrix shear strength. Similarly, $Y_{m t}$ and $Y_{m c}$ are determined by applying global transverse stresses $\bar{\sigma}_{22}=Y_{t}$ and $Y_{c}$. Equations (46) and (47) are then solved with $d_{m}=1, \beta=1$, and $\gamma=2$ for the matrix axial strengths in tension and compression. Finally, $X_{f t}$ and $X_{f c}$ are obtained by subjecting the RVE to global axial stresses $\bar{\sigma}_{11}=X_{t}$ and $X_{c}$. The ensuing axial stresses in the fiber subcell, $\bar{\sigma}_{11}^{(11)}$ are used for maximum allowable fiber stresses in tension and compression.

If any of Equations $(46),(47),(48)$, or (49) are met in any matrix subcell, all the subcell properties are reduced by $99 \%$. As in method 1 , once failure has occurred in any subcell, progressive damage is deactivated 


\begin{tabular}{|l|l|}
\hline Property & Value (Msi) \\
\hline \hline$Y_{m t}$ & 0.00656 \\
\hline$Y_{m c}$ & 0.0183 \\
\hline$T_{m}$ & 0.0059 \\
\hline$X_{f t}$ & 0.758 \\
\hline$X_{f c}$ & 0.414 \\
\hline
\end{tabular}

Table 7. Constituent failure strengths.

at that integration point.

\section{Results and Discussion}

Load versus edge displacement data is reported for all three laminates using both models (ST/H-R and $\mathrm{ST} / \mathrm{GMC} / \mathrm{T}-\mathrm{H}$ ) and compared to experimental results. Additionally, applied local strains are compared to experimental results obtained from four strain gages placed at the locations shown in Figure 6(a). The elements used to represent these strain gages are shown in Figures 6(b) and 6(c). Data in these elements are averaged to obtain the strain data at that strain gage location. Table 8 contains landmark experimental and computational loads in each of the laminates, such as splitting and ultimate loads.

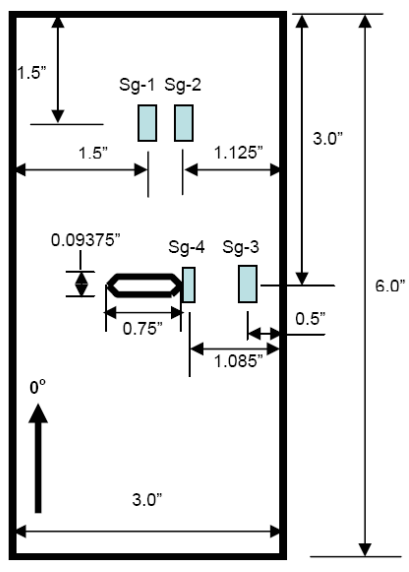

(a) Strain gage locations in experiment. ${ }^{5}$

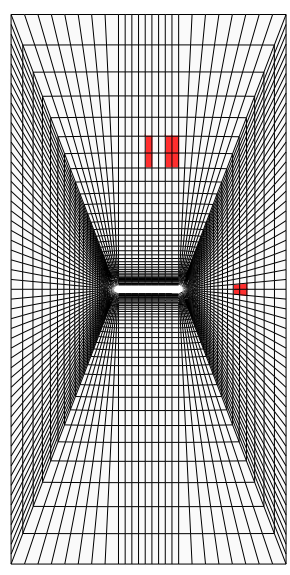

(b) Elements used to represent strain gages in simulation.

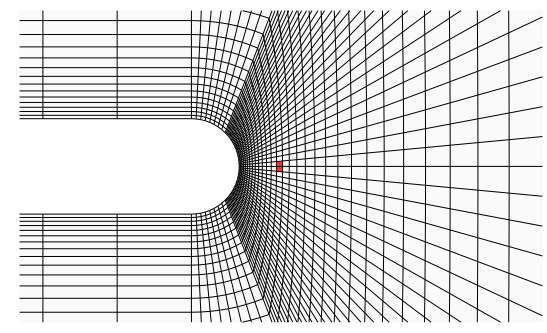

(c) Elements used to represent Sg-4 in simulation.

Figure 6. 


\begin{tabular}{|l|l|c|c|c|}
\hline Laminate & Load Type & Experiment & ST/H-R & ST/GMC/T-H \\
\hline \hline Laminate 1 & Splitting & 8635 lbs. & 8600 lbs. & 8792 lbs. \\
\hline Laminate 2 & Ultimate & 12632 lbs. & 9780 lbs. & $12322 \mathrm{lbs}$. \\
\hline
\end{tabular}

Table 8. Critical Loads.

\section{IV.A. Laminate Sequence-1}

Applied load versus the edge displacement (of a 4 inch section) results from both techniques are compared to experimental data in Figure 7. It can be seen that the multiscale method, ST/GMC/T-H, yields data that is

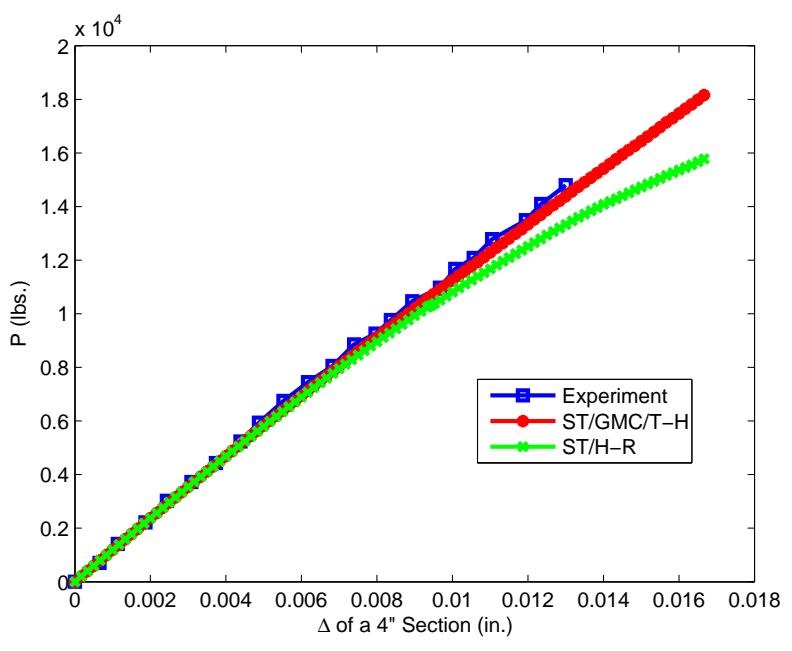

Figure 7. Load versus displacement of a 4" section, laminate sequence 1.

extremely close to experiment. The load/displacement response obtained using ST/H-R are still desirable. Although the curve exhibits softening not present in the experiment or in the other model, this softening is well after the experimental splitting load, which was reported to be $8635 \mathrm{lbs}$. in Ref. 5, and may be a product of the semi-gradual manner in which the properties of the failed elements are reduced.

Far field load versus local strain data is presented for all four gages in Figure 8. The splitting load predicted by both simulations can be extracted from the sg- 1 data (Figure $8(\mathrm{a})$ ). ST/GMC/T-H predicts a splitting load of $8600 \mathrm{lbs}$., and ST/H-R anticipates a splitting load equal to $8792 \mathrm{lbs}$. These values are reported in Table 8. Localized hardening can be observed in the results. This hardening is actually localized strain relaxation at sg-1 and is a consequence of failure near the notch. The results for both simulations at sg-2 (Figure 8(b)) exhibit error. This could be because of the large element size at that location; so, no integration point is very near the actual strain gage location. The data at sg-4 using both approaches also displays some variation from the experimental data. This is to be expected, however, because the strain gradients near the notch tips are extremely high.

The failure patterns produced by ST/GMC/T-H and ST/H-R are shown in Figure 9. Matrix failure is represented in the $\mathrm{ST} / \mathrm{H}-\mathrm{R}$ results with green elements, and fiber failure is indicated with red elements. The $\mathrm{ST} / \mathrm{GMC} / \mathrm{T}-\mathrm{H}$ results represent 1 matrix subcell failure with turquoise elements, 2 failed matrix subcells with green elements, 3 failed matrix subcells with yellow elements, 1 failed fiber subcell and 3 failed matrix subcells (4 total) with red elements.

The matrix failure patterns generated from both simulations are in agreement with each other and represent matrix shear splitting exhibited in experiment (Figure 10). The two methods produce different fiber failure patterns. At the onset of fiber failure, stiffness contributions due to the matrix near the notch are severely diminished. However, it is possible that some of the matrix in some elements near the notch is still intact. Additionally, initial fiber failure can further contribute to the variations in stiffnesses near the notch tip. This gradient in material properties can lead the notch tip to enter a bifurcated state (Figure 11(b)), which in turn drastically affects the stress distribution in the surrounding region. This a facet of the simulation and is not occurring in the experiment because the material is actual opening at the notch tip 


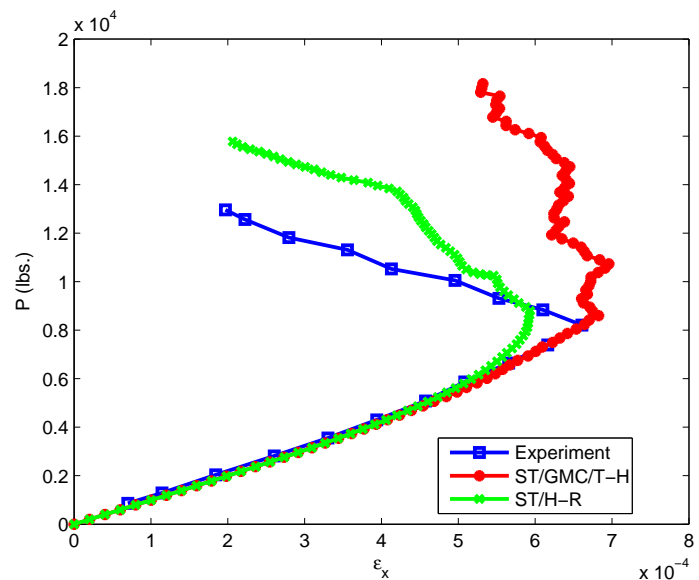

(a) $\mathrm{sg}-1$

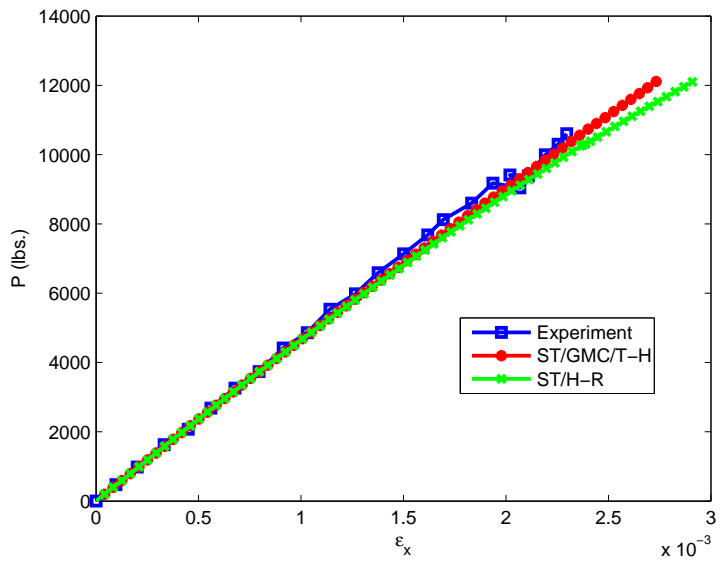

(c) $\mathrm{sg}-3$

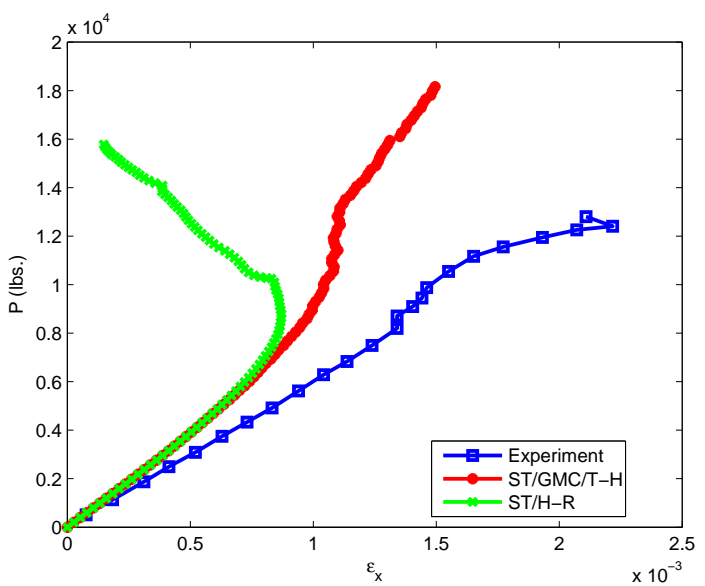

(b) sg-2

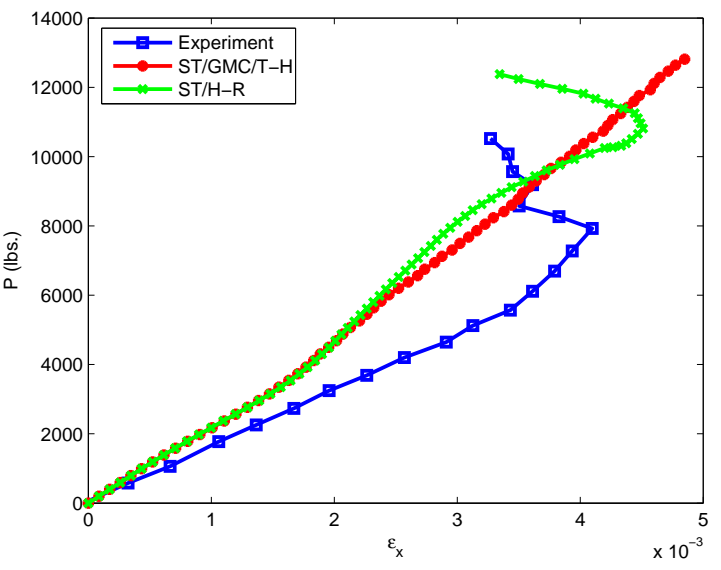

(d) sg-4

Figure 8. Load vs. strain for Laminate 1. 


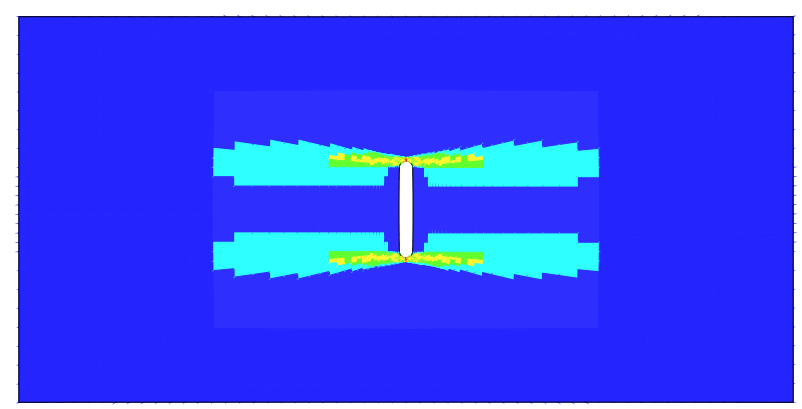

(a) ST/GMC/T-H failure pattern, $\mathrm{P}=14048 \mathrm{lbs}$.

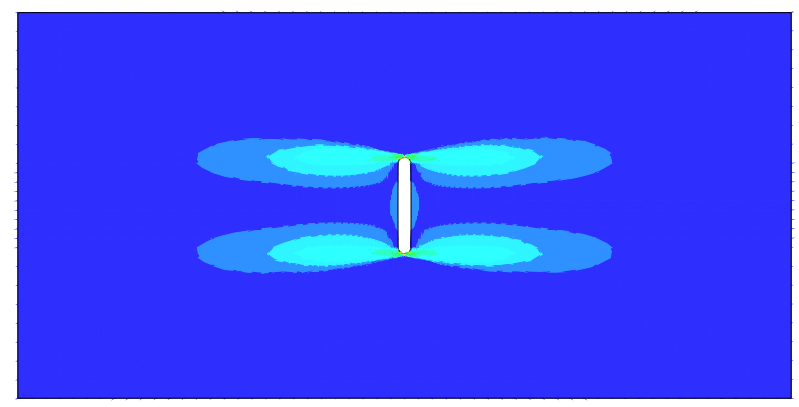

(c) ST/GMC/T-H damage pattern, $\mathrm{P}=8600 \mathrm{lbs}$.

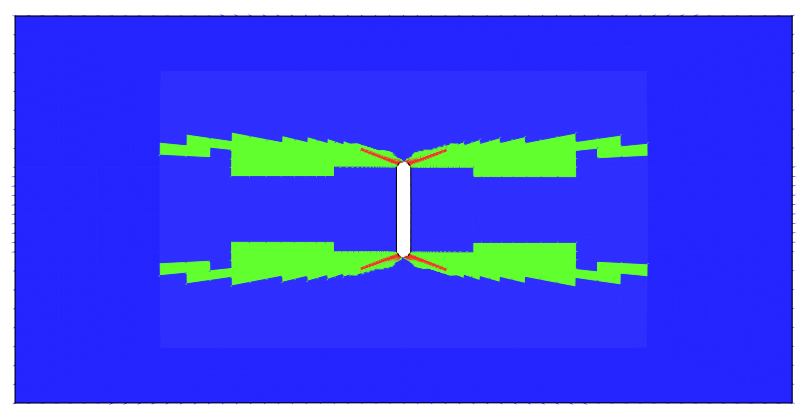

(b) ST/H-R failure pattern, $\mathrm{P}=14082 \mathrm{lbs}$.

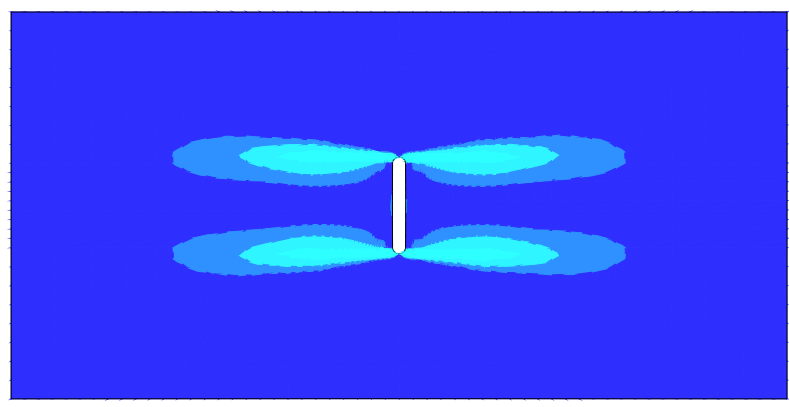

(d) ST/H-R damage pattern, $\mathrm{P}=8792 \mathrm{lbs}$.

Figure 9. Failure and damage paths in $0^{\circ}$ layer of Laminate 1.

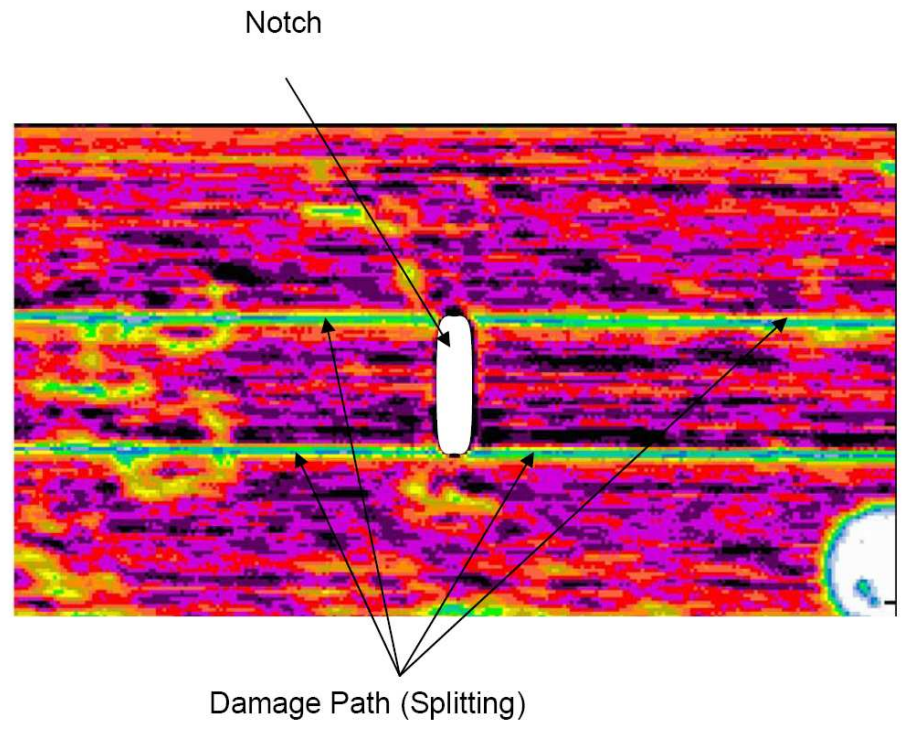

Figure 10. C-Scan of failed laminate 1 specimen exhibiting splitting. ${ }^{5}$ 


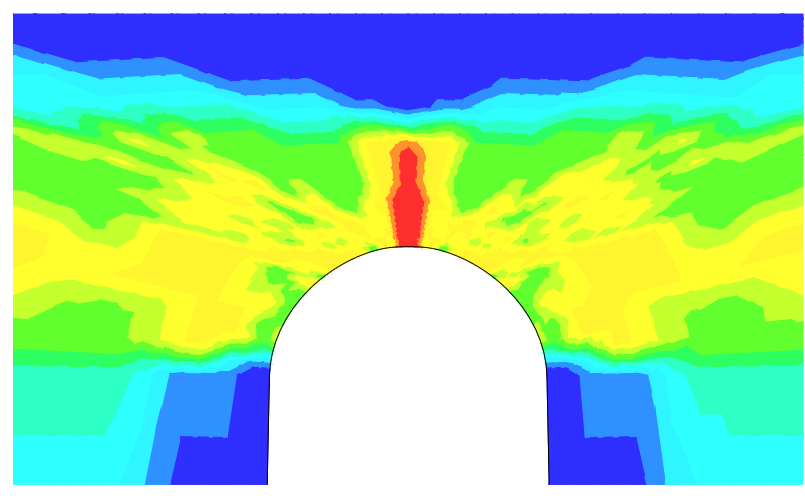

(a) $\mathrm{ST} / \mathrm{GMC} / \mathrm{T}-\mathrm{H}$

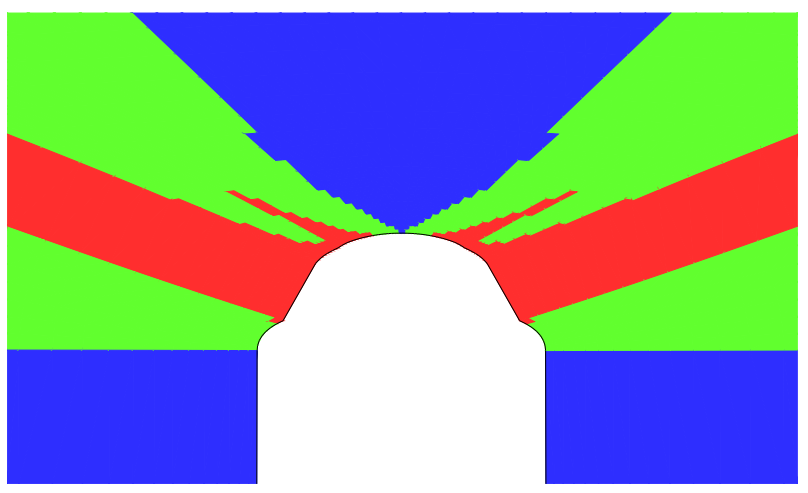

(b) ST/H-R

Figure 11. Magnification of failure in laminate 1 at notch tip.

and in the split regions. Additionally this behavior is highly sensitive to the ratio of the matrix strengths, and displays the highly coupled nature of failure mechanisms in composite materials. Thus, the fiber failure displayed by ST/H-R is not physical. The fiber failure path produced by ST/GMC/T-H is the expected mechanism (Figure 11(a)).

Figure 9 also shows the microdamage patterns calculated using both methods. These damage patterns are similar, and they progress in the same manner as the matrix failure shown in Figures 9(b) and 9(a). This indicates that matrix failure in $0^{\circ}$ laminates is a culmination of microdamage.

\section{IV.B. Laminate Sequence-2}

Figure 12 shows the bulk response for laminate sequence-2 and Figure 13 show the local strain gage data for both simulations and experiment. The ultimate load prediction from ST/H-R (12322 lbs.) is far more

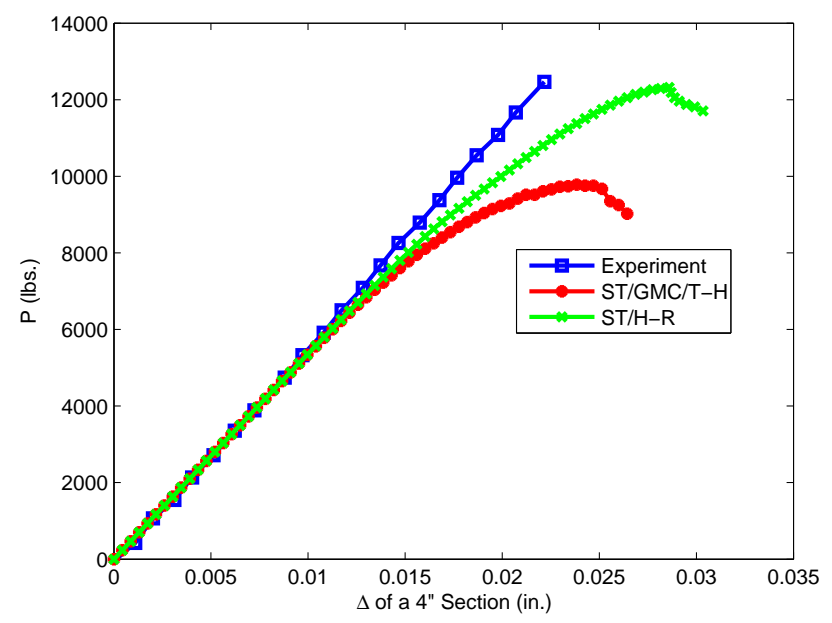

Figure 12. Load versus displacement of a 4" section, laminate sequence 2 .

accurate than from ST/GMC/T-H (9780 lbs.) when compared to the reported experimental ultimate load (12632 lbs.). Since fiber failure is dictating the ultimate load in this laminate, the failure criterion used at the fiber level in ST/GMC/T-H needs to be revisited, and re-formulated in terms of a critical fiber strain to failure to obtain more consistent results.

The failure paths for the $+45^{\circ}$ layer are displayed in Figure 14 and use the same convention as the previous laminate. The failure patterns for the top layer closely represent the failure mechanisms observed in experiment (Figure 15). However, there is failure mechanism present in the ST/H-R simulation not present in the multiscale calculations. The laminate modeled using ST/H-R experienced fiber failure, due 


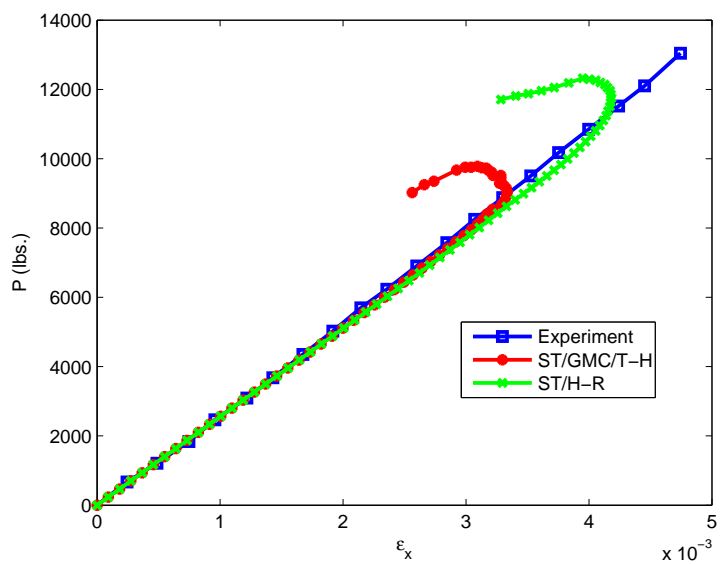

(a) $\mathrm{sg}-1$

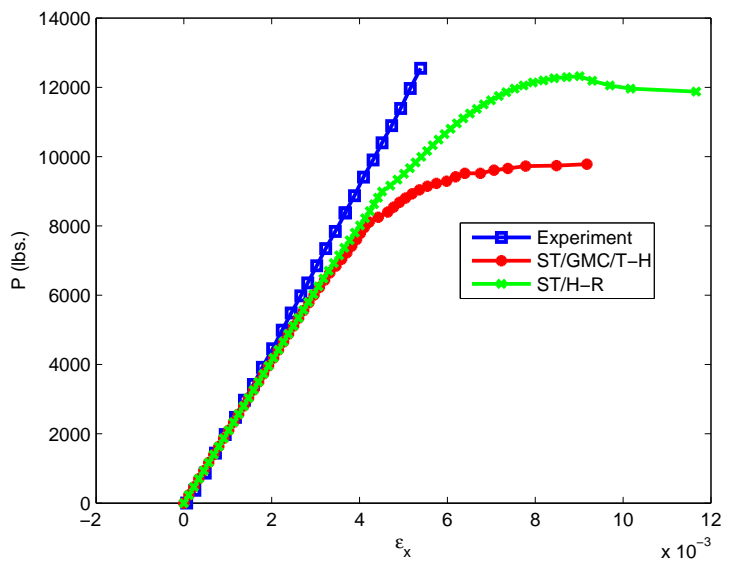

(c) $\mathrm{sg}-3$

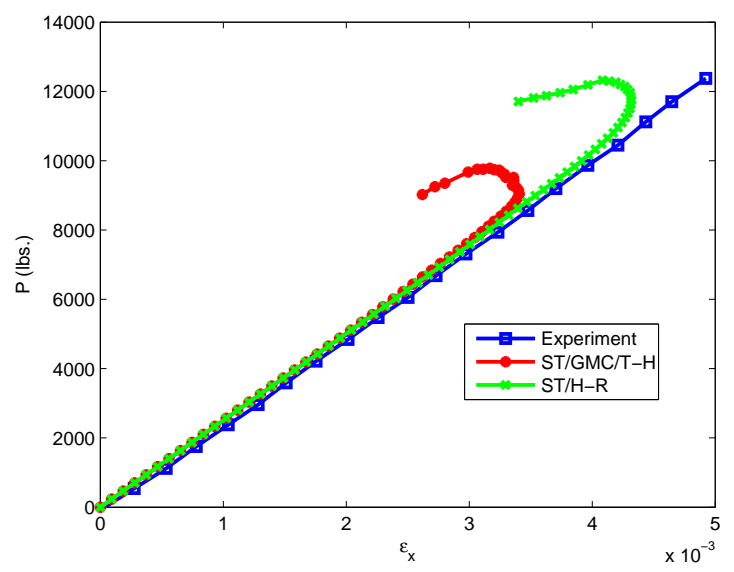

(b) sg-2

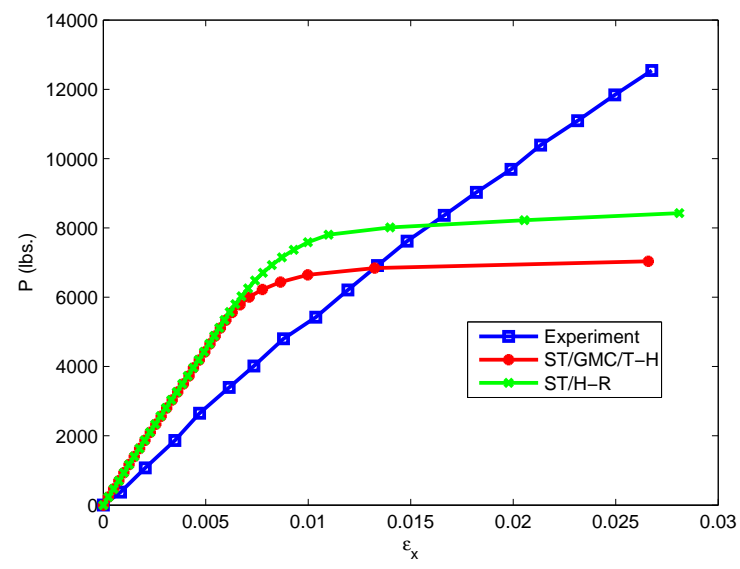

(d) sg-4

Figure 13. Load vs. strain for Laminate 2. 


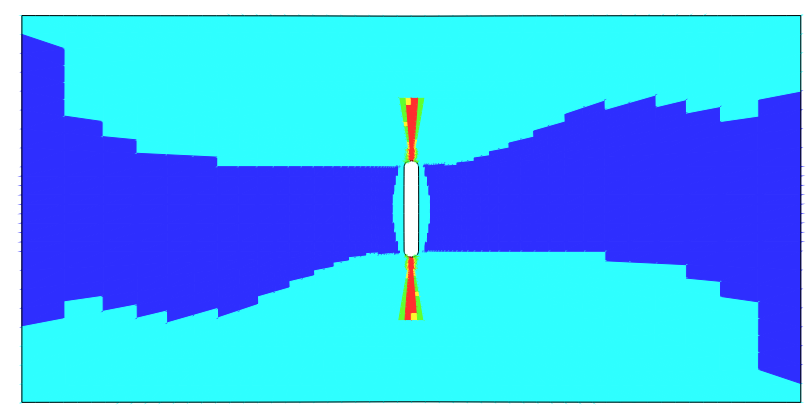

(a) ST/GMC/T-H failure pattern, $\mathrm{P}=9788 \mathrm{lbs}$.

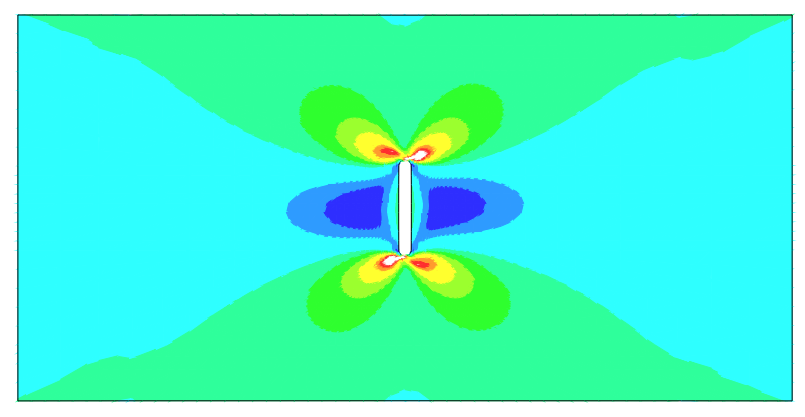

(c) ST/GMC/T-H damage pattern, $\mathrm{P}=4878 \mathrm{lbs}$.

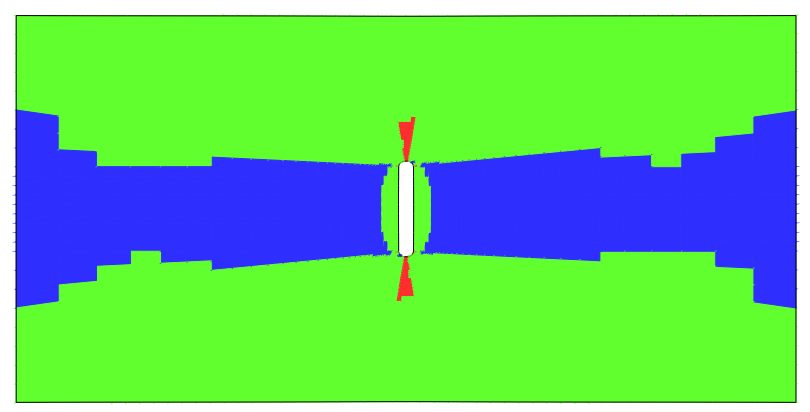

(b) $\mathrm{ST} / \mathrm{H}-\mathrm{R}$ failure pattern, $\mathrm{P}=12322 \mathrm{lbs}$.

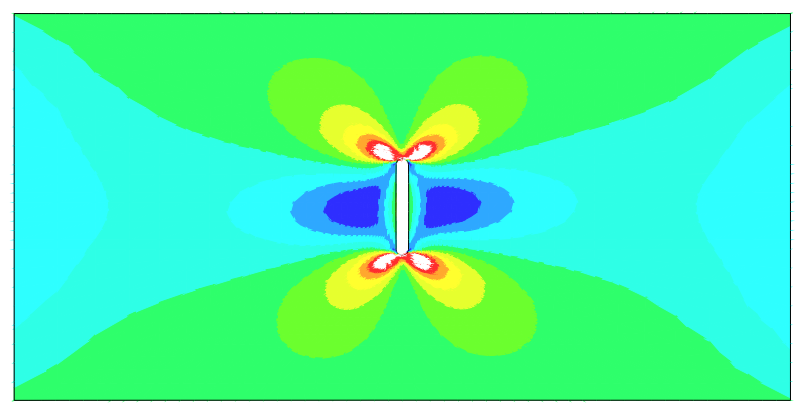

(d) ST/H-R damage pattern, $\mathrm{P}=5572 \mathrm{lbs}$.

Figure 14. Failure and damage paths in $45^{\circ}$ layer of Laminate 2.

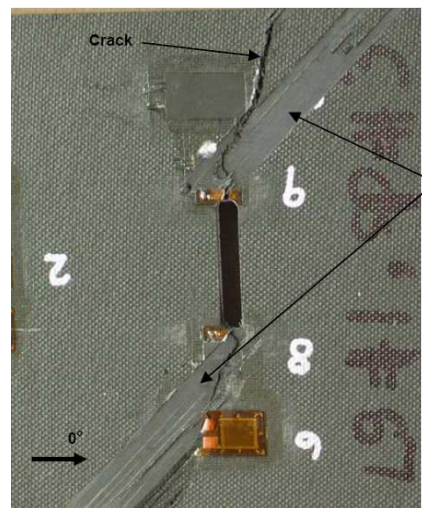

Figure 15. Photograph of failed Laminate 2 specimen. ${ }^{5}$ 
to compression at the point where the notch radius meets the slot, see Figure 16. The activation of this

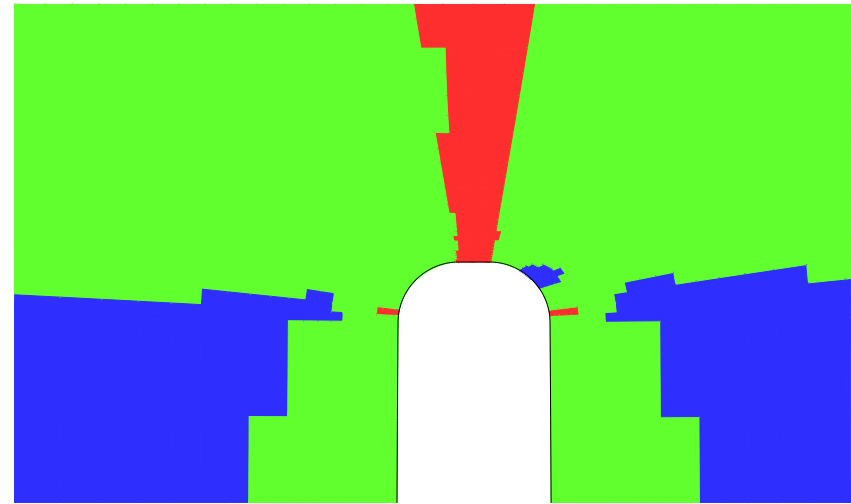

Figure 16. Compressive fiber failure in $45^{\circ}$ layer of laminate 2 .

failure mechanism dissipates energy and allows the laminate to reach a higher ultimate load. Figure 17 shows the failure patterns in the $0^{\circ}$ and $90^{\circ}$ plies. The presence of compressive fiber failure in this laminate

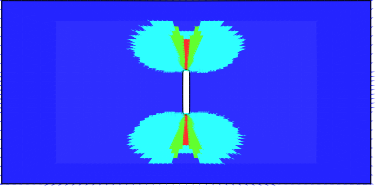

(a) ST/GMC/T-H failure pattern in $0^{\circ}$ layer.

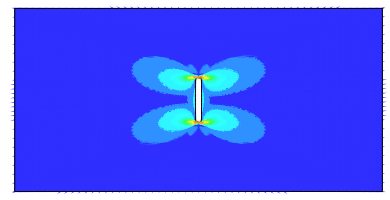

(e) ST/GMC/T-H damage pattern in $0^{\circ}$ layer.

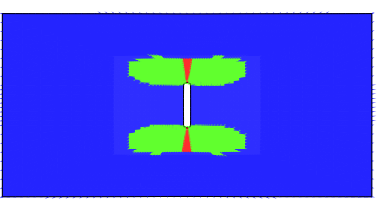

(b) ST/H-R failure pattern in $0^{\circ}$ layer.

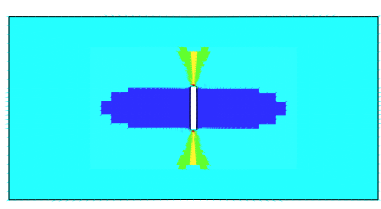

(c) $\mathrm{ST} / \mathrm{GMC} / \mathrm{T}-\mathrm{H}$ failure pattern in $90^{\circ}$ layer.

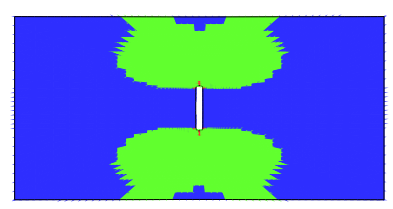

(d) ST/H-R failure pattern in $90^{\circ}$ layer.

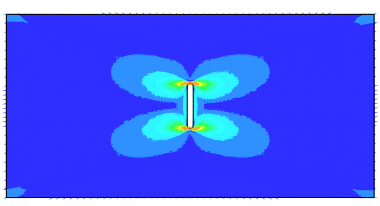

(f) $\mathrm{ST} / \mathrm{H}-\mathrm{R}$ damage pattern in $0^{\circ}$ layer.

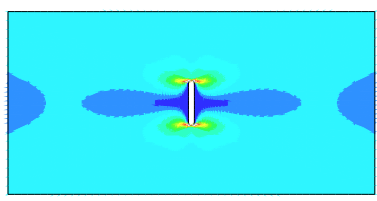

(g) $\quad \mathrm{ST} / \mathrm{GMC} / \mathrm{T}-\mathrm{H}$ damage pattern in $90^{\circ}$ layer.

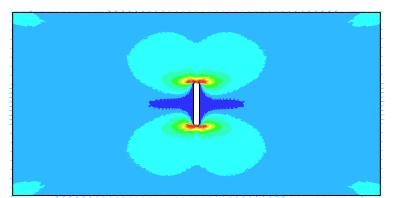

(h) ST/H-R damage pattern in $90^{\circ}$ layer.

Figure 17. Failure and damage paths in $0^{\circ}$ and $90^{\circ}$ layers of Laminate 2.

demonstrates that incorporating fiber rotation $^{8}$ in the progressive damage calculations is warranted, even though the specimens are loaded in tension. An added advantage of this would be the ability to dispose of a failure criterion to predict compressive fiber failure by setting a limit on the amount of allowable fiber rotation or rate of fiber rotation. Both simulations exhibit the same matrix splitting and fiber failure in the $0^{\circ}$ plies and an extensive amount of matrix damage in the $90^{\circ}$ plies.

Microdamage patterns, produced far before the accumulation of failed elements is significant, show patterns similar to the matrix failure patterns produced when the laminate has reached its ultimate load. The microdamage patterns for all layers are given in Figures 14 and 17. This indicates that matrix failure in this laminate is a product of microdamage and may be characterized by incorporating a critical limit on the ISV, $S_{r}$, rather than using a macroscopic failure criterion for the matrix.

The use of constant failure strengths may be influencing the results of laminate 2. Matrix shear strength, $T$, governs the failure behavior in the $0^{\circ}$ lamina. Since this layer carries more load than any other ply in the the multi-angle laminate, the ultimate loads of the laminate is dictated by the performance of this lamina. However, $T$ and $T_{m}$ are calibrated using laminate sequence-1. At the onset of matrix failure, the failed elements have already damaged progressively; hence, the material at the location of matrix failure is 
different than the material in the far field. Furthermore, the level of progressive damage near the notch in all layers of laminate 2 is different than that experienced in laminate 1; therefore, the material properties at those locations will also be different. Using the same shear strength for all laminates is analogous to predicting failure in different materials with the same shear failure constant. This argument can be extended to the other failure constants influencing matrix failure $\left(Y_{t}, Y_{c}, Y_{m t}\right.$, and $\left.Y_{m c}\right)$; although the influence of these parameters on the bulk response of the laminates is not as profound as $T$ or $T_{m}$.

Test results for a third laminate sequence were reported in Bogert et al. ${ }^{5}$ However, in this third sequence, significant delaminations were observed, and it was reported that progressive failure in the laminate occurred simultaneously with delamination failure. In the present work delamination as a failure mechanism is not modeled. A model for delamination, however, is presented in Ref. 16.

\section{Conclusion}

Progressive matrix microdamage in a uniaxially loaded center notched specimen has been modeled using Schapery Theory (ST). However, the damage modes associated with progressive damage are separate from those caused by catastrophic failure. Thus, it is necessary to develop a criterion that evolves progressive damage into catastrophic failure. Two novel methods for capturing both progressive damage and failure were presented. One uses lamina level ST and lamina level Hashin-Rotem (H-R) failure theory (ST/H-R). The other incorporates lamina level ST with failure evaluated at the micro-constituent level using the TsaiHill (T-H) failure criterion for the matrix and a maximum stress criterion for the fiber $(\mathrm{ST} / \mathrm{GMC} / \mathrm{T}-\mathrm{H})$. Failure strengths used in these criteria were calibrated against experimental results from a $[0]_{12}$ T800/3800-2 laminate.

The performance of these methods were then evaluated on a multi-angle laminate $\left([+45 / 0 /-45 / 0 / 90]_{S}\right)$ of the same material. Qualitative predictions from ST/T-H were accurate. The ultimate load was underpredicted by ST/GMC/T-H. Since fiber failure is the failure mechanism that most influences the ultimate load, it is inferred that the maximum stress criterion is not desirable in a multiscale method. A maximum strain criterion may provide better results. The failure path predictions from both methods represented the observed experimental failure modes accurately. Additionally the progressive damage patterns mirrored the matrix failure patterns determined using a matrix failure criterion. Thus, this criterion may be redundant, and it may be possible to eliminate the matrix failure criterion altogether by determining a critical damage state, evolved from the progressive ST damage model. Additionally, fiber failure in compression is controlled by fiber rotation, which is a product of the stiffness in the surrounding matrix. Therefore, this failure mechanism can also be predicted using progressive damage, as shown earlier in Ref. 8. Since, fiber failure in tension is not a progressive phenomenon, a method to account for fiber breakage in tension is still needed. Therefore, this study has shown that accurate modeling of the progressive degradation of the matrix, together with a method to account for fiber direction failure in tension and a critical evolved damage state are sufficient to completely capture in-plane failure of FRLs.

\section{Acknowledgements}

The authors appreciate the interest and support of Philip Bogert, Alex Tessler, and Arunkumar Satyanarayana at NASA Langely Research Center. This work was supported through a NASA contract to Collier Research Corporation. 


\section{References}

${ }^{1}$ Schapery, R.A. (1990). A theory of mechanical behaviour of elastic media with growing damage and other changes in structure. J. Mech. Phys. Solids, 38(2), 215-253.

${ }^{2}$ Hashin, Z., and Rotem. A. (1973). A fatigue failure criterion for fiber reinforced composite materials.J. Composite Materials. Vol 7. 448-464.

${ }^{3}$ Paley, M., and Aboudi J. (1992). Micromechanical analysis of composites by the generalized cells model. Mechanics of Materials, 14(2), 127-139.

${ }^{4}$ Jones, R.M. (1999). Mechanics of Composite Materials, 2nd Ed.. Taylor and Francis, Inc., Philadelphia.

${ }^{5}$ Bogert, P.B., Satyanarayana, A., and Chunchu, P.B. (2006). Comparison of damage path predicions for composite laminates by explicit and standard finite element analysis tool. $47^{\text {th }}$ AIAA Structures, Structural Dynamics, and Materials Conference, 1-4 May, Newport, Rhode Island.

${ }^{6}$ Sicking, D.L. (1992). Mechanical characterization of nonlinear laminated composites with transverse crack growth. Ph.D. Theses, Texas A\&M University, College Station, Texas.

${ }^{7}$ Rice, J.R. (1971). Inelastic constitutive relations for solids: an internal-variable theory and its application to metal plasticity. J. Mech. Phys. Solids, 19(6), 433-455.

${ }^{8}$ Basu, S., Waas, A.M., and Ambur, D.R. (2006). Compressive failure of fiber composites under multiaxial loading. Int. J. Solids and Structures, 44(9), 2648-2676.

${ }^{9}$ Aboudi, J. (1991). Mechanics of Composite Materials: A Unified Micromechanical Approach. Elsevier Amsterdam.

${ }^{10}$ Aboudi, J., Pindera, M.J., and Arnold, S.M. (1999). Higher-order theory for functionally graded materials. Composites Part B - Engineering, 30(8), 777-832.

${ }^{11}$ Pineda, E.J., Waas, A.M., et al. (2007). A mesh independent implementation of laminate failure criteria. in preparation

${ }^{12}$ Bhargava, A., and Shivakumar, K.N. (2007). Three dimensional tensila stress concentration in countersunk rivet holes. Aeronautical Journal, Volume 111, Issue 1126, 777-786

${ }^{13}$ A BAQUS User's Manual. (2003). Vol 1-3, Version 6.5, Hibbit, Karlsson, and Sorenson, Pawtucket, RI.

${ }^{14}$ Bednarcyk, B.A., and Arnold, S.M. (2002). MAC/GMC 4.0 User's Manual - Keywords Manual,NASA/TM 2002212077 /VOL2

${ }^{15}$ Bednarcyk, B.A., and Arnold, S.M. (2002). MAC/GMC 4.0 User's Manual - Example Problems Manual,NASA/TM $2002-212077$ /VOL3.

${ }^{16}$ Satyanarayana, A. Bogert, P.B., Chunchu, P.B. (2007). The effect of delamination on damage path and failure load prediction for notched composite laminates. $48^{\text {th }}$ AIAA Structures, Structural Dynamics, and Materials Conference, $23-26$ April, Honolulu, Hawaii. 